[RADiOCARbon, Vol. 21, No. 1, 1978, P. 22-40]

\title{
DESIGN, CONSTRUCTION AND CALIBRATION OF \\ A HIGH ACCURACY CARBON-14 COUNTING SET UP
}

\author{
$P$ P TANS and W G MOOK \\ Physics Laboratory, Department of Isotope Physics, \\ University of Groningen \\ Westersingel 34, 9718 CMI, Groningen, 'The Netherlands
}

ABSTRACT. The construction and performance of a proportional $\mathrm{CO}_{22}$ counter system is described by which an over-all accuracy is obtained of $1.3 \%$ after a two-day counting period.

\section{INTRODUCTION}

Natural fluctuations of atmospheric ${ }^{1 .} \mathrm{C}$ on time scales of a clecade to a century, as established by the ${ }^{11} \mathrm{C}$ measurement of tree rings, have a magnitude of a few permil to a few percent, while the recent dilution of ${ }^{14} \mathrm{C}$ by the combustion of fossil fuels also amounts to a few percent.

In order to make the fullest possible use of the ${ }^{14} \mathrm{C}$ record, the accuracy of measurement, which usually is $4-5 \%$, should be improved, preferably to about $1 \%$, in fact, a technical improvement by a factor of 20 . Apart from the geophysical implication, such accurate measurements can be used for the dendrochronologic calibration of the ${ }^{14} \mathrm{C}$ time scale. Also, floating tree chronologies from different parts of the world can be anchored firmly in time by "wiggle matching", provided the wiggles are well identified (De Jong, Becker, and Mook, 1979).

\section{CHOICE OF COUNTING GAS}

A statistical precision of $1 \%$, (one standard deviation) is attained by the accumulation of a million counts. If this has to be accomplished in two one-day counting periods, the counter has to contain two moles of carbon, as the specific disintegration rate of carbon-14 in modern material is $13.5 \mathrm{dpm} / \mathrm{g}$ C. Two moles of carbon are equivalent to $50 \mathrm{~L}$ NTP of $\mathrm{CO}$. gas. In order to avoid enormous counter volumes we have to choose a higher than usual gas pressure.

Use of a different gas, such as ethane, reduces the above requirement considerably as more carbon atoms are being stored in one molecule of gas. In all instances, the sample is first converted into $\mathrm{CO}$., by combustion. The use of a counting gas like ethane, however, requires a more elaborate sample preparation procedure.

The additional steps would introduce an open-system procedure, with greater danger of contamination. A principal draw-back of the ethane preparation would also be a possible production of higher hydrocarbons which introduces an uncertainty in the amount of carbon brought into the counter. Therefore, we decicled to adhere to our $\mathrm{CO}$. tradition. The sensitivity of $\mathrm{CO}$., to electronegative impurities in the counter does not pose a serious problem, if the combustion is accompanied by sufficient purification stages. 

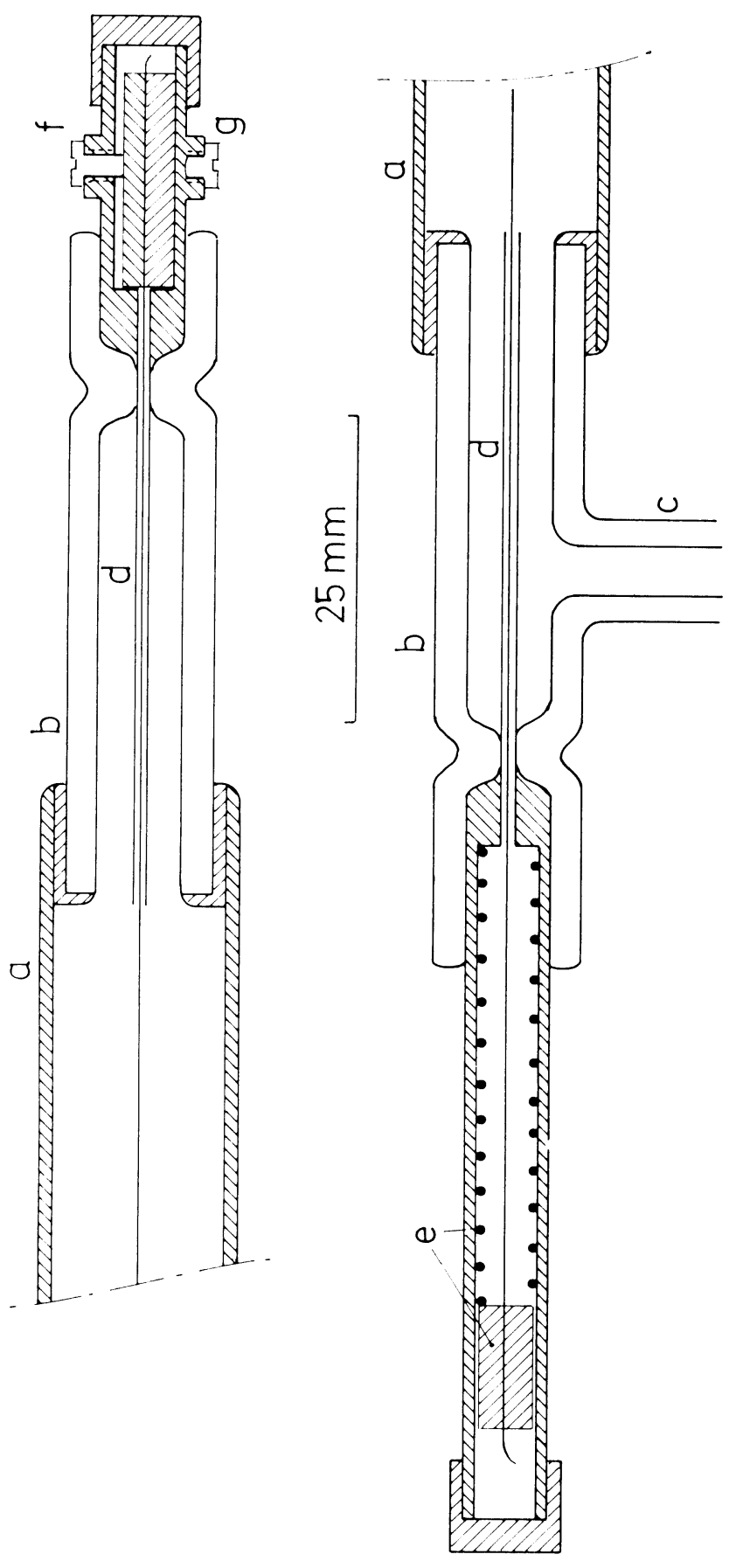

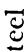

क

葛

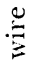

के

華

$\stackrel{\Xi}{\Xi}$

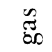

e

تั)

芯

N $\widehat{2}$

ฮั

ठ

$\prod$ नृत्त

氖

过

๖े

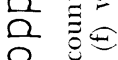

O

N $\tilde{0} \cdot \frac{\pi}{2}$

$\cong$

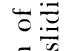

苛

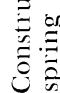

-

$\stackrel{\ddot{g}}{\underline{G}}$ 
A PRELIMINARY EXPERIMENT WITH HIGH GAS PRESSURES

The large amount of gas needed inevitably leads to the use of high pressures of the counting gas. In order to investigate the properties of $\mathrm{CO}_{2}$ as a counting gas, such as the amplification and the sensitivity for electronegative impurities (Brenninkmeyer and Mook, 1979) at these pressures, a small counter was constructed for use at pressures up to $16 \mathrm{~atm}$. The construction was kept as simple as possible (fig. 1).

Cosmic ray muon events were minimized by an anticoincidence arrangement using a cylinder of plastic scintillator that surrounded the working volume of the counter. The scintillator was viewed from both ends by two photomultiplier tubes working in coincidence. The distance between the scintillator and the photomultipliers was bridged by cylindric perspex lightguides surrounding the counter endpieces and fitted with holes to accommodate the gas inlet and the signal and high-voltage wires. The shielding consisted of $10 \mathrm{~cm}$ of lead bricks. Measurements have been performed up to pressures of $16 \mathrm{~atm}$. In practice, a limit on the pressure is posed by the gas purity resulting from a standard treatment on the combustion system. The results were used to calculate the optimal dimensions of the high-precision counting system.

The absolute determination of the gas multiplication factor was based on the relative variation of the high voltage, the gas pressure and the puls height discriminator level (Groeneveld, 1977). The muon counting rates are determined as a function of the high voltage for a number of pressures and discriminator settings. It has been shown from general considerations (Rossi and Staub, 1949) that the gas amplification can be expressed as

$$
\frac{\ln M}{a E_{a}}=f\left(\frac{E_{a}}{p}\right)
$$

where $M$ is the amplification factor, $a$ is the radius of the central wire, $E_{a}$

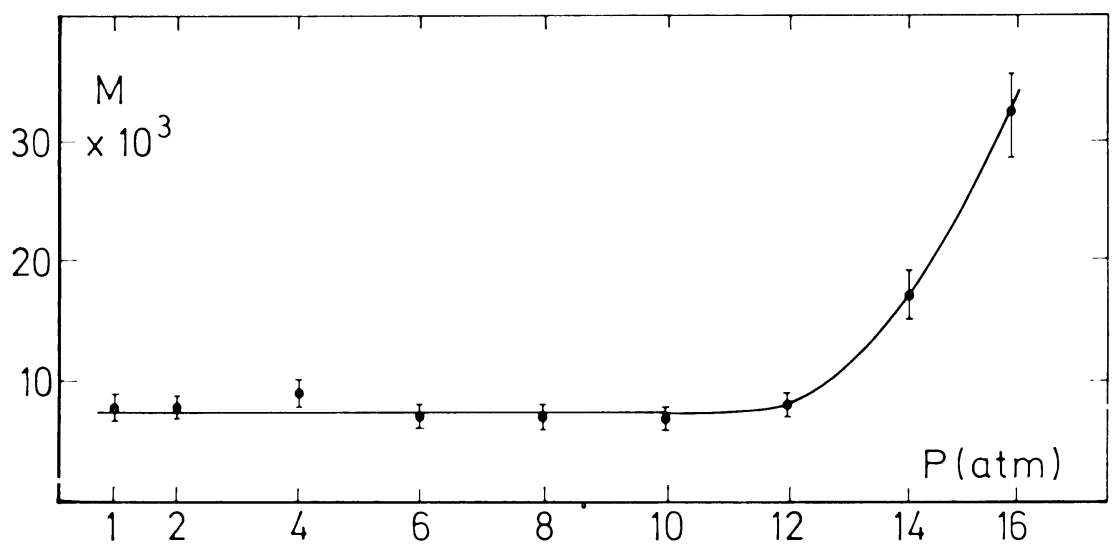

Fig. 2. Gas amplification needed to surmount a fixed level of pulse height discrimination with a certain number of primary electrons. This series of measurements has been carried out with the same, highly purified gas sample, starting at the highest pressure and letting the gas escape stepwise. 
the electric field strength at the wire surface, and $p$ the gas pressure. Using Groeneveld's method, a curve of $(\ln M) / a E_{a}$ against $E_{a} / p$ is constructed that enables us to calculate the gas amplification for all counter dimensions, voltages, and pressures. Our values nicely fitted a similar curve of Grootes (1977) up to pressures of 12 atm. Above this pressure the calculated amplification that is needed to pass a fixed discriminator level increases for ${ }^{14} \mathrm{C}$ in a drastic manner (fig. 2).

The capture of electrons by electronegative impurities has adequately been clescribed as a three-body process (Herzenberg, 1969). The rate of loss of electrons by attachment to an impurity like $\mathrm{O}_{2 .}$, depends on the concentration of both $\mathrm{O}_{2}$ and $\mathrm{CO}_{2}$. The original excited $\mathrm{O}_{2}$, caused by the electron attachment, releases the electron after a short lifetime $(5 \times$ $10^{-12} \mathrm{~s}$, Spence and Schulz, 1972) unless the ${\mathrm{O} .2^{-*}}^{-*}$ is stabilized by colliding with a $\mathrm{CO}_{2}$ molecule within this time span. If we are dealing with a gas with a constant impurity fraction, Brenninkmeyer and Mook (1979) have shown that the increase in gas multiplication required to offset the loss of primary electrons by attachment obeys the relation

$$
\ln \frac{M}{M_{o}}=\frac{c^{2} f K p^{3} b^{2} \ln b / a}{2 \mu V}
$$

where $a$ and $b$ are the radii (in $\mathrm{cm}$ ) of the central wire and the counter respectively, $V$ is the high-voltage applied, $p$ is the filling pressure (in torr), $f$ is the impurity fraction (in ppm), and $c^{2} / 2 \mu \cong 10^{21}$; the attachment coefficient $K$ is a constant, depending on the nature of the trace gases $\left(\mathrm{O}_{2,}, \mathrm{SO}_{2}, \mathrm{NO}_{2}, \mathrm{H}_{2} \mathrm{O}\right)$. Since, in our case, $\mathrm{ln} M$ was found to be more nearly proportional to $p^{1.5} / V$, some process other than this three-body attachment seems to be effective. Without further investigation of this effect we decided to keep the $\mathrm{CO}$. pressures below $10 \mathrm{~atm}$.

In practice, even the limit of $10 \mathrm{~atm}$ is not within reach as already at lower pressures the effect of impurities will severely hamper the performance of the counter. Therefore, we also studied the highest tolerable level of electron attachment with the small counter. Predetermined amounts of $\mathrm{O}_{2}$, have been added to a previously purified sample. The resulting shift and distortion of the characteristic curve is shown in figure 3 .

The distortion is due to the fact that primary electrons originating close to the wall suffer more electron attachment than those formed near the wire. Both the 1 and 5 ppm curves at 12 atm and the 5 ppm curve at $6 \mathrm{~atm}$ are unacceptable, as they do not provide a stable working point. The uncertainty in the purity correction to be applied becomes too large.

The question now is what these curves mean in terms of pulse height loss. For both pressures, the voltage at which the gas amplification equals 8000 has been calculated. At these voltage levels, the percentage of primary electrons starting at the counter wall and reaching the wire has been calculated, using eq 2. For the $1 \mathrm{ppm}$ and the $5 \mathrm{ppm}$ curve at 6 atm and the 1 ppm curve at 12 atm these fractions are 78, 29, and 26 percent respectively. It should be noted that $p^{3} / V$ differs by a factor of 5.5 for both working voltages chosen, so that 1 ppm $\mathrm{O}_{2}$ at $12 \mathrm{~atm}$ 
should indeed have a slightly larger effect than $5 \mathrm{ppm}$ at $6 \mathrm{~atm}$. Our knowledge about the impurity correction does not allow a correction to be applied for impure samples showing this primary electron loss to be more than 30 percent, leading to a decreased counting rate of several percent. This corresponds to a loss of primary ionization electrons that is three times as high as the amount of loss calculated for other counters in operation at our laboratory. As they leave the combustion system, the samples usually have a lower apparent $\mathrm{O}_{2}$ concentration (equivalent to a maximum primary electron loss of 5 percent).

\section{DESIGN AND CONSTRUCTION OF THE HIGH-ACCURACY SYSTEM}

General considerations.-In designing the detection system the error due to the counting statistics of the ${ }^{14} \mathrm{C}$ beta decay should be the only error significantly influencing the accuracy of the measurement. Errors in temperature, pressure, and time measurements, and in the background and impurity correction should be an order of magnitude smaller. A low background is desirable as it allows the detection of instabilities more easily.

The amount of counter filling is about $50 \mathrm{~L}$ STP. The maximum working length, $80 \mathrm{~cm}$, is limited by the length of the available GM anticoincident counters. In our laboratory, other ${ }^{14} \mathrm{C}$ counters, with a working voltage of $7000 \mathrm{~V}$, have no detectable spurious pulses due to electrical leakage across the insulators, provided they are kept clean and dry. At a gas amplification factor of 8000 , the counter(s) are operated on the counting plateau.

With the above limitations, the wire diameter needed to achieve a gas amplification of 8000 has been calculated for several possible counter configurations and gas pressures by using the experimentally determined curve of $(\ln M) / a E_{a}$ versus $E_{a} / p$. Subsequently, the loss of primary electrons between the counter wall and the wire was derived for an $\mathrm{O}_{2}$ concentration of $0.2 \mathrm{ppm}$, thus providing an estimate for the severity of the impurity effect (Tans, 1978).

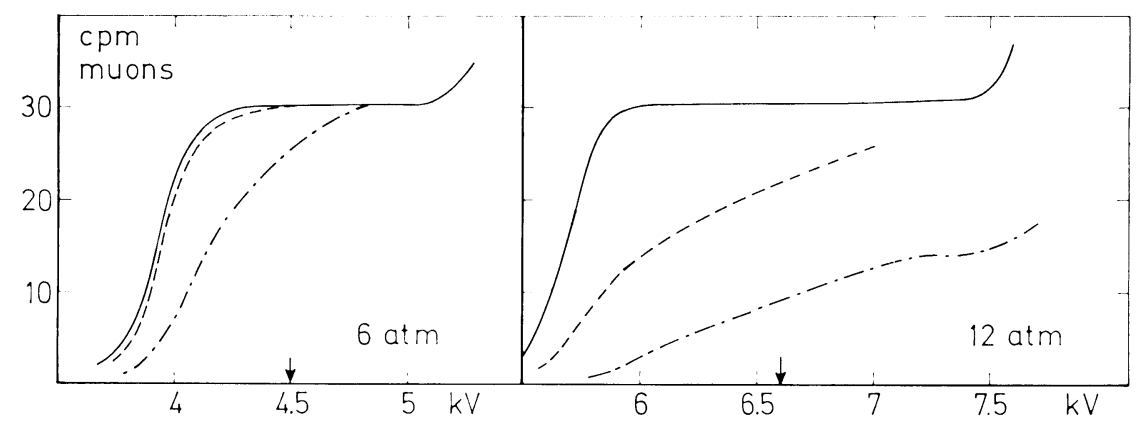

Fig. 3. Muon counting characteristics. The effect of small amounts of oxygen on the counter performance. The arrows indicate the voltages at which the gas amplification factor equals 8000 . Curves are presented for pure gas (solid line) and gas containing 1 ppm of $\mathrm{O}_{2}$ (dashed line) and 5 ppm of $\mathrm{O}_{2}$ (dash-point line). 
It is not possible to operate satisfactorily a large counter at high pressures because of the effect of the impurities. From those configurations that could meet our 70 percent criterion for the impurity effect we have chosen the system consisting of 7 counters operating at $6 \mathrm{~atm}$, as it provides the smallest overall diameter, which is important for attaining a low background.

High-purity quartz is the ideal material for our counters. It is radiochemically pure, and the counters can be isolated electrically from each other by applying a conductive coating to both the inside and outside. The inside is connected to a negative high voltage, while the outside is grounded. If the thickness of the quartz tube is small, the counter wall, itself, has a large capacity (in our case $3 \mathrm{nF}$ for each counter). Together with the resistance that is embodied in the high-voltage connecting plug
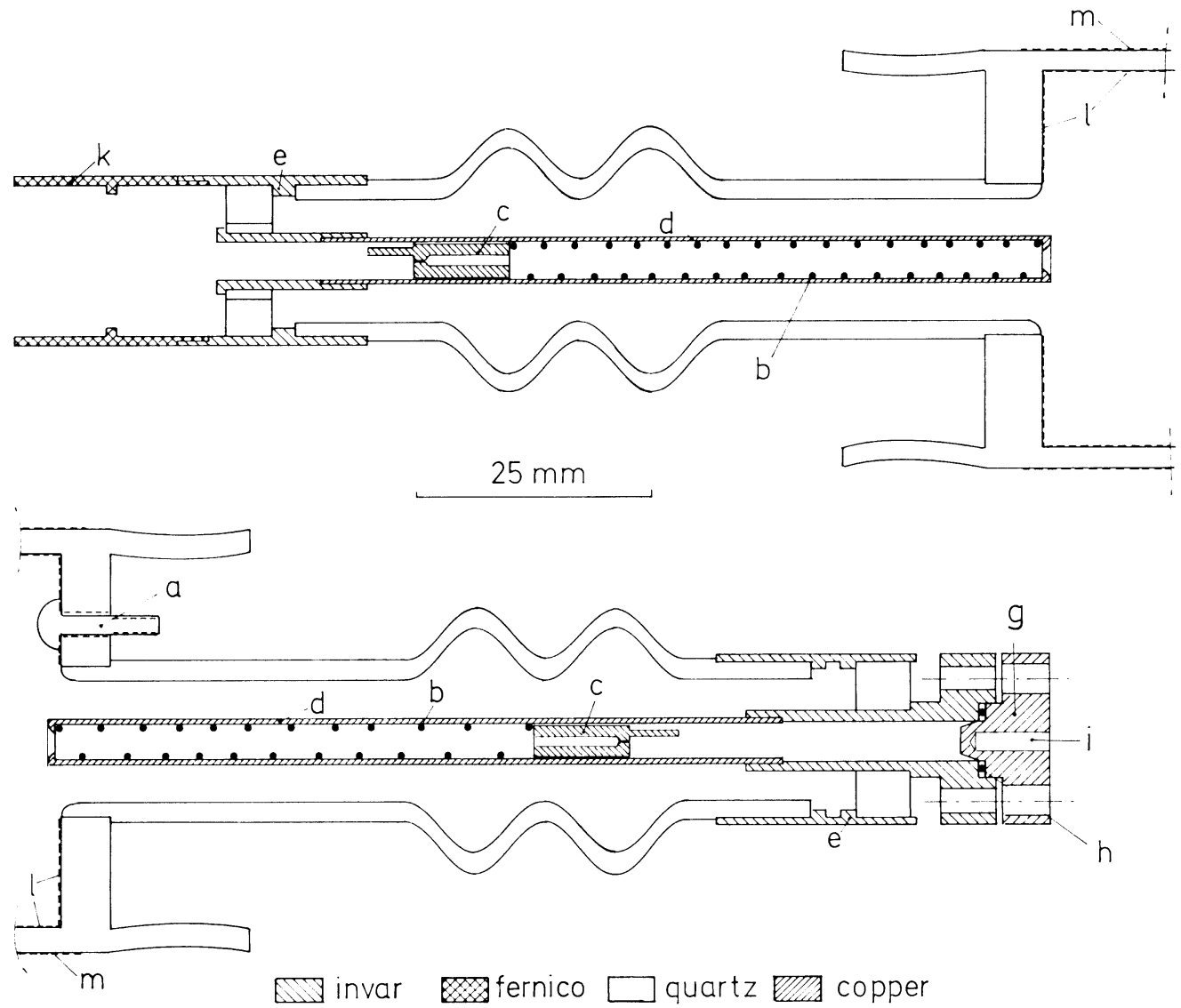

Fig. 4. Design of the end pieces of the counters. (a) high voltage connection; (b) spring for ten sioning of the central anode wire; (c) moving piston to which the wire is attached; (d) wire support; (c) guard rings; (g) removable end plug; (h) gold ring; (i) socket for connection to the preamplifier; (k) fernico (54 percent $\mathrm{Fe}, 29$ percent $\mathrm{Ni}, 17$ percent $\mathrm{Co}$ ), to match the thermal characteristics of the pyrex gas inlet: (l) conductive layer of $0.01 \mu \mathrm{m}$ of gold; (m) conductive layer, silver paint. 
it serves as a high-voltage integrating filter. In this way, spurious pulses caused by leakage of charge from the internal lead shield to the external counter wall are avoided.

Construction and installation of the counter system.-The design of the endpieces is shown in figure 4. The quartz end flanges have been melted into the counter tube. The cleaning operations, the vacuum deposition of the gold film, and the assembly have been performed as described by Grootes (1977). However, a two-component epoxy, curing at $80^{\circ} \mathrm{C}$, has been used. On one side, the counters are closed by a gold ring allowing to fit a new wire without taking apart any epoxy seal.

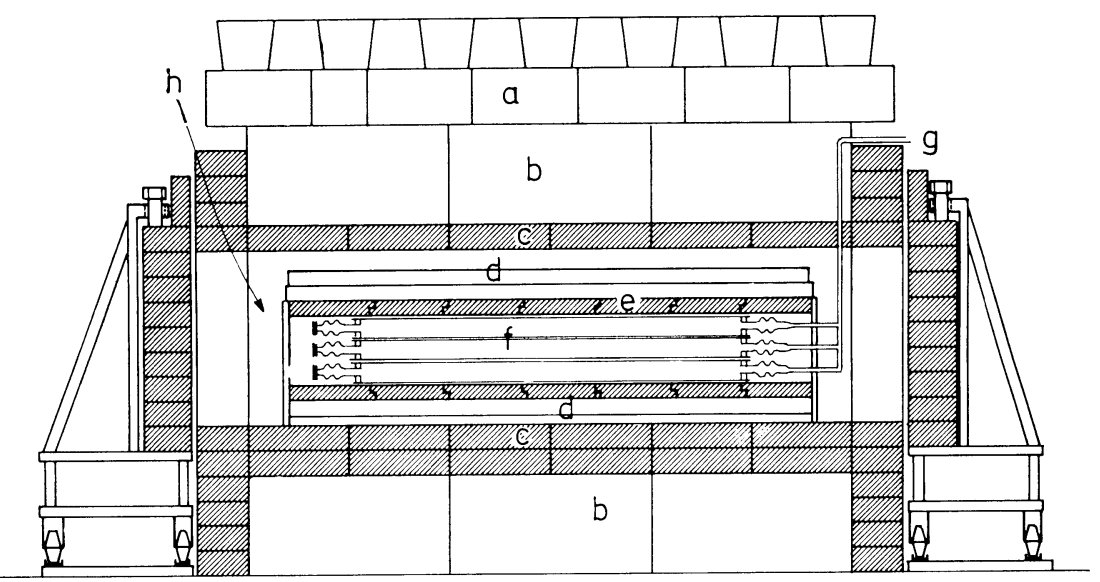

$50 \mathrm{~cm}$

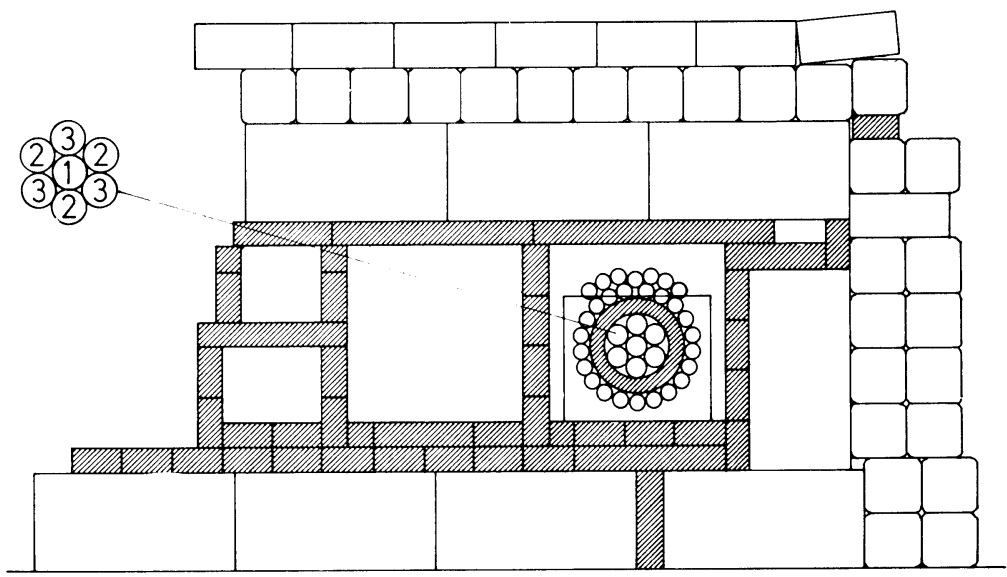

Fig. 5. Counter arrangement and shiclding. (a) iron; (b) boron-paraffine; (c) lead; (d) Geiger-Müller anti-coincidence counters; (c) old lead; (f) proportional counters; (g) gas inlet; (h) electrical connections. Proportional counters with equal numbers are connected in parallel to the same preamplifier and can be operated as one separate counter. 
The gas inlet connection is made by using two pyrex ball-joints pressed together by aluminium flanges. The viton O-rings of the joints do not deteriorate the gas purity. As a result, the removal or replacement of a counter is very simple.

The counters are installed in a concentric arrangement (fig. 5A and B) inside a cylinder of specially selected old lead with a thickness of $2.5 \mathrm{~cm}$. They are made accessible by a front and a rear door of $10 \mathrm{~cm}$ of lead on rail. The underground laboratory has a ceiling consisting of 1-m soil and concrete.

For reasons of electrical insulation, the temperature of the inner lead cylinder is kept a few degrees above room temperature. The temperature of the counters is measured by a platinum resistance thermometer with a reproducibility of $0.01^{\circ} \mathrm{C}$ (Doric DS-100). The platinum sensor is placed halfway between the counters.

The counters are connected such that, as an option, a sample can be admitted to only the central counter or to one of both sets of three surrounding counters (equal numbers in fig. 5B). In order to protect the counter wires and the electronics, the gas supply lines have been provided with pressure-sensitive switches that shut off the high-voltage power supply, if the pressure falls below a preset level.

Pressure measurement is by a conventional diaphragm manometer (Philips PCS transmitter D). The pressure is referred to a reference pressure of $5.697 \mathrm{~atm}$, the vapor pressure of propylene at a temperature of $0.00^{\circ} \mathrm{C}$. The reproducibility of the manometer is 0.001 times the total range of $0.5 \mathrm{~atm}$. The reproducibility of the pressure measurement is, therefore, determined by the degree of control on the temperature of the water-ice mixture in which a propylene flask is immersed (fig. 6). An $0.01^{\circ} \mathrm{C}$ variation of this thermostat temperature is equivalent to an apparent change of the specific activity of the sample of $0.3 \%$. The temperature of the water-ice mixture is being monitored continuously with a platinum temperature sensor. The temperature variations are generally less than $0.01^{\circ} \mathrm{C}$. The samples are stored at high pressure $(\sim 60 \mathrm{~atm})$ in stainless steel containers. Filling of the counters proceeds by a controlled expansion from the storage bottles directly into the counters. It has been checked by ${ }^{13} \mathrm{C}$ measurements that within experimental limits of $\pm 0.1 \%$ no isotopic fractionation occurs during this process, the time of expansion having been varied between a few minutes and a quarter of a minute. The valves of the storage bottles are manufactured completely from metal parts in order to preserve the purity of the gas during storage.

Signal processing.-The pulse-shaping networks, the preamplifiers, and amplifiers are located inside the shielding. The signal processing is depicted schematically in figure 7 . The central counter and each set of three counters are not only operated in anti-coincidence with the GM counters but also with the other proportional counters. The pulses are separated into a high and a low-energy channel. The information obtained from the relative counting rates of both channels is used to determine a correction to the total counting rate due to differences in gas 


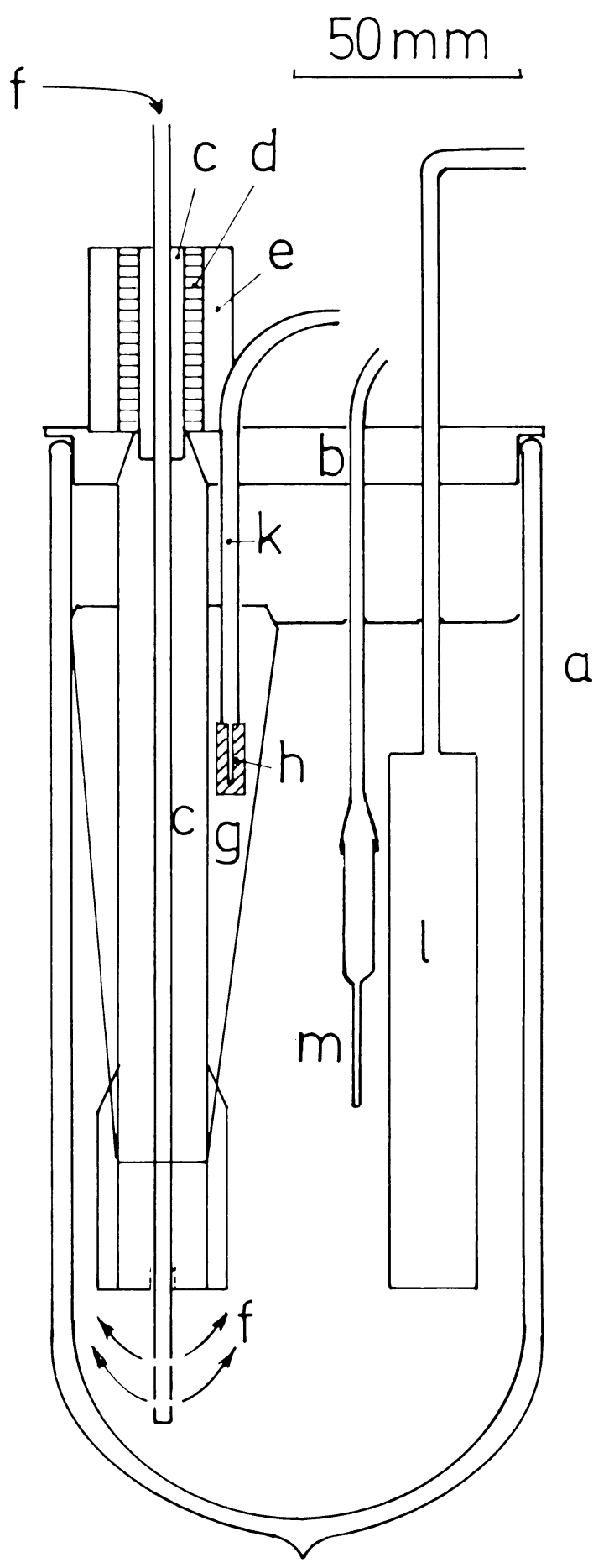




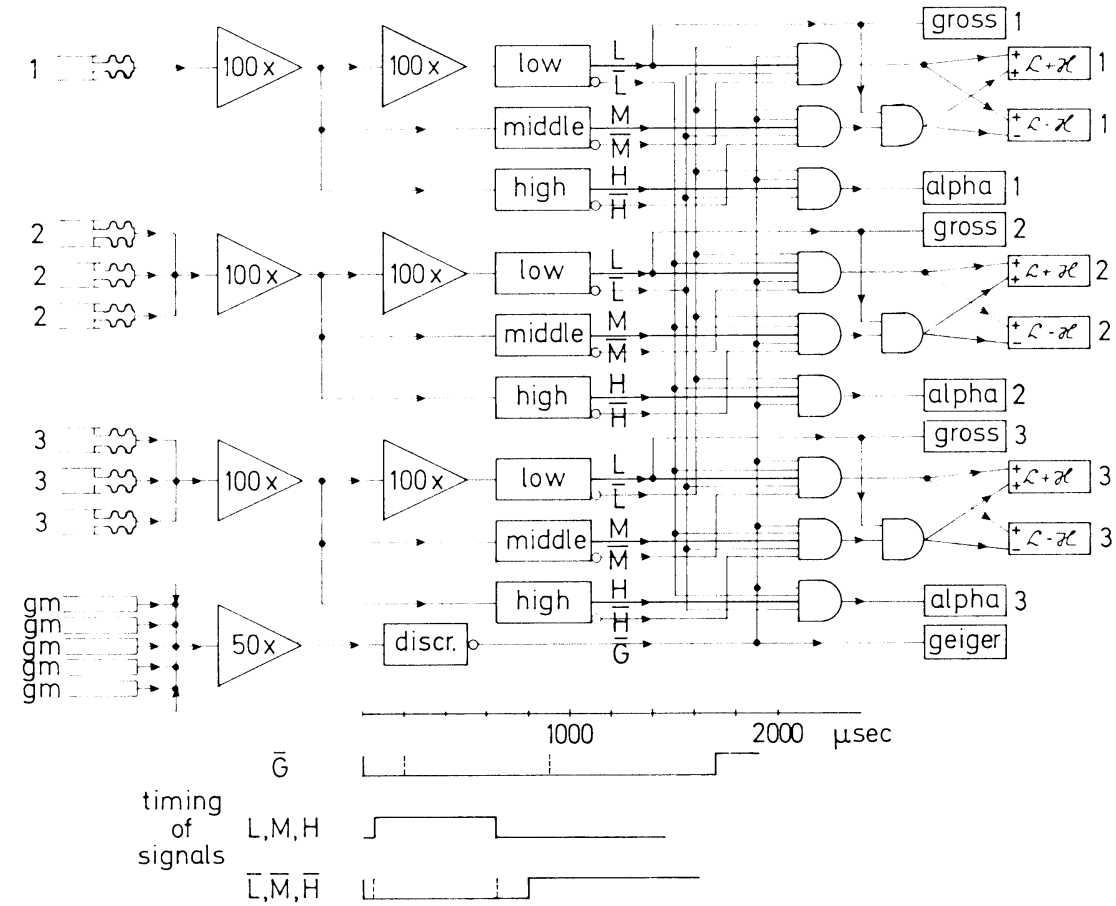

Fig. 7 . Scheme of signal processing. The final counting channels are: grossmuons + beta's + background + alpha's; $L+H$ total ${ }^{14} \mathrm{C}$ beta + background; $L-H$ purity channel of ${ }^{14} \mathrm{C}$ beta + background; alpha, pulses above the maximum ${ }^{14} \mathrm{C}$ decay energy.

purity. The discriminators after the first amplifier define the separation between both channels and the upper threshold of the high-energy channel. The discriminator after the second amplifier defines the low threshold of the low-energy channel. The pulses from both channels are fed to up-down counters, one of them summing the channels $(\mathrm{L}+H)$, the other subtracting the counting rate of the high-energy channel from that of the lower one $(L-H)$. In order to minimize the likelihood of pick-up in the cables connecting the inside of the shielding to the discriminators, pulses in the high-energy channel are accepted only if they have also been seen in the low channel. This increases the threshold for pick-up to the level of the low discriminator (at $5 \mathrm{~V}$ ). Great care was taken that the electronic system did not contain ground loops.

« Fig. 6. Thermostat bath for the reference pressure vessel. (a) dewar; (b) cover, perspex; (c) heat exchanger, copper; (d) peltier cooling elements; (e) flow chamber for water cooling; (f) stream of dried air for stirring; (g) layer of ice; (h) thick walled copper tube with thermistor inside; (k) thin walled tube for thermistor leads; (l) propylene vessel; $(\mathrm{m})$ platinum resistance temperature sensor. The manometer reference side and the immersed vessel are filled with $\sim 12$ atm of propylene. The thermostat bath is cooled to $0^{\circ} \mathrm{C}$ and the propylene partly condenses on the walls of the immersed vessel. The thermistor regulates the power supply to the peltier batteries. If the layer of ice becomes too thick, the batteries are switched to a lower power level. 
The relative position of the low and the middle discriminator thresholds is important for the stability of the system and the accuracy of the purity correction. The counters are always operated at the same high voltage which is chosen so that the gas amplification produces a pulse height spectrum cut in half by the middle discriminator. In other words, the operating voltage is chosen halfway the steep part of the highenergy channel counting characteristic. A small deterioration of gas purity then causes a relatively large shift of the spectrum into the lower channel. At the same time, the low discriminator threshold should

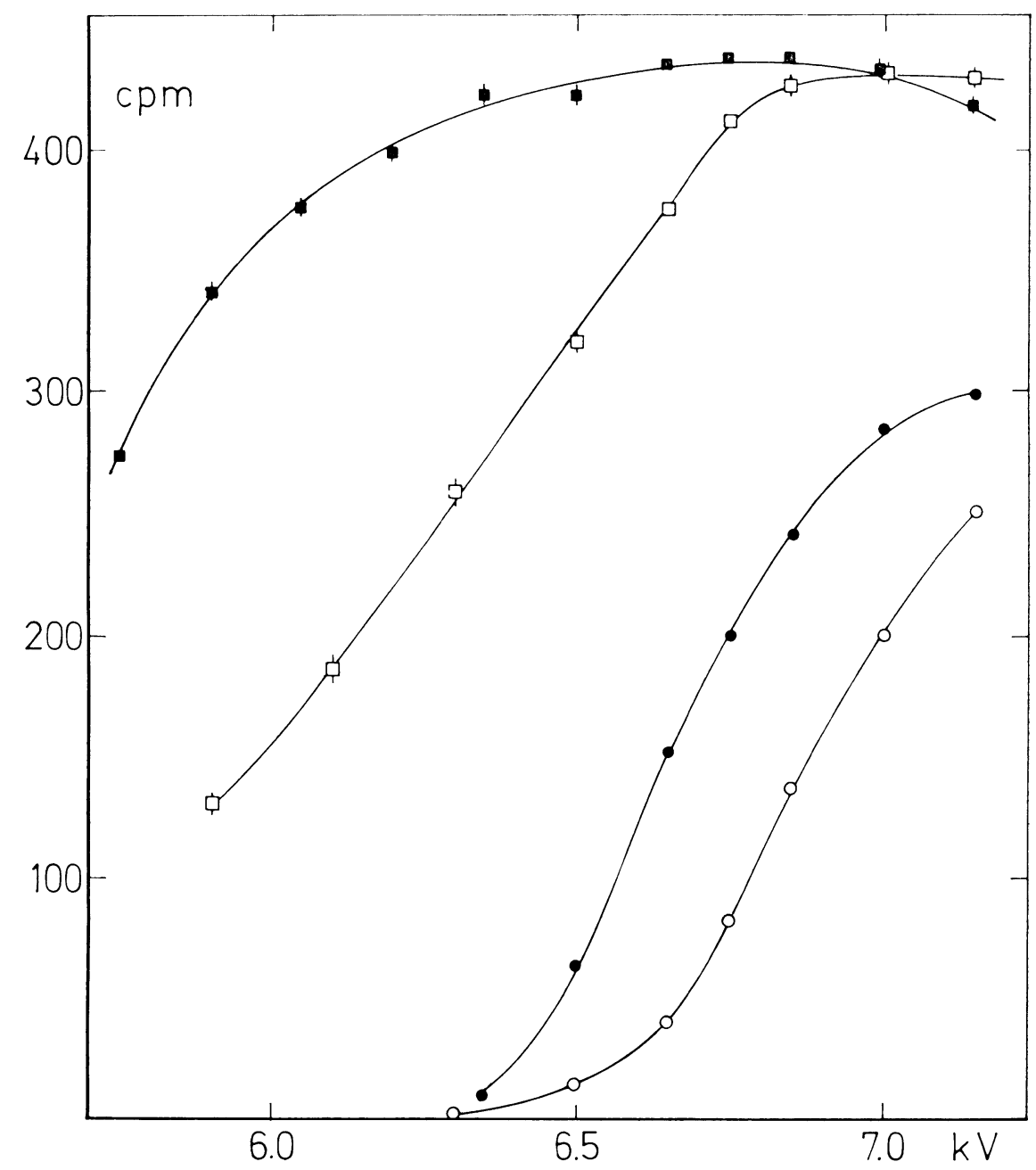

Fig. $8 .{ }^{14} \mathrm{C}$ counting characteristics with and without an artificial impurity (oxygen). The sample is 1963 oak wood. Squares, ${ }^{14} \mathrm{C}$ from both channels combined; circles, only the high energy channel; open and solid symbols, with and without additional oxygen (1.5 ppm), respectively. 
be low enough that in this case few pulses only are lost from the total counting rate. Drift of the preamplifier is seen by the system as an apparent change in gas purity for which it is, to first order, automatically corrected. This correction procedure is not perfect as a real change in gas purity slightly influences the shape of the spectrum, whereas amplifier drift does not. The same is true for a drift of equal sign and of equal proportions simultaneously occurring to all thresholds. However, changes of the middle discriminator level and in the amplification of the second amplifier alone are interpreted by the system as variations in gas purity, and a correction to the total counting rate will then be applied. Drift of the low threshold alone results in real changes of the counting rate. The discriminator settings have been chosen such that a 5 percent drift of one of the last three factors would result in a $1 \%$ change of the counting rate.

The long-term stability (over a period of a year or more) is not relevant as all measurements are referred to a reference sample 0.95 times the activity of NBS oxalic acid, which is measured regularly.

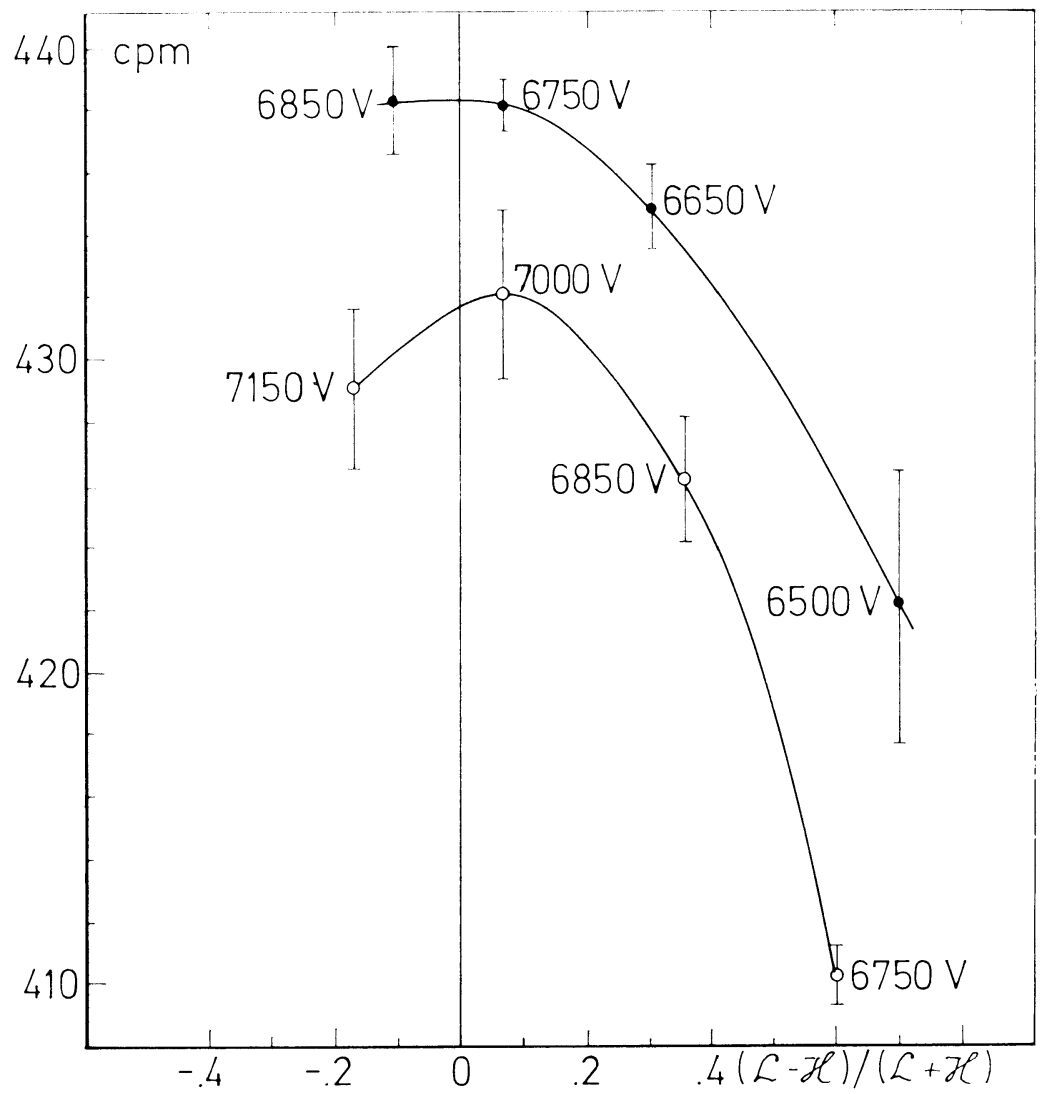

Fig. 9. The counting rate as a function of the relative purity, as defined by $(L-H) /(L+H)$. Open and solid circles, with and without additional oxygen (1.5 ppm). An increase of the high voltage does not entirely restore the counting rate to the value for high-purity gas. 
PERFORMANCE OF THE COUNTER SYSTEM

${ }^{14} \mathrm{C}$ counting characteristics.-Only the calibration of the entire system is presented. Figure 8 shows part of the counting characteristics of the sum of both ${ }^{14} \mathrm{C}$ channels as well as of the high-energy channel alone. The working voltage is $6750 \mathrm{~V}$, at which the gas amplification is calculated to be 9500 . Also shown in figure 8 , is the effect of additional $O_{2,}$ at a concentration of about $1.5 \mathrm{ppm}$. The distortion of the counting characteristic is rather large due to the fairly large amount of $\mathrm{O}_{22}$ added. The necessity of a fully empirical purity correction clearly emerges from figure 9 , where the total ${ }^{14} \mathrm{C}$ counting rate has been plotted against the relative purity counting rate $(L-H) /(L+H)$. Nerely a parallel displacement of the characteristic curve should have put both curves of figure 9 on top of each other. The empirical correction was determined by measuring oxalic acid for various levels of gas purity (fig. 10). The power curve fitted to these measurements is actually used for the purity correction of all samples. Below a relative impurity $(L-H) /(L+H)$ of 0.25 , the type of function fitted hardly affected the purity correction. Samples above 0.25 have to be rejected for direct measurement and are to be repurified. In practice, however, our wood samples provide $\mathrm{CO}_{2}$ showing $(L-H) /$ $(L+H)$ values below 0.05 .

The error due to the uncertainty of the purity correction (maximum $0.4 \%$ of the total net counting rate at present) has become smaller after additional oxalic acid measurements with different gas purities have become available.

Background.-The dependence of the background on the counter gas pressure is +4 percent/atm. This is of the same order of magnitude as has been found for other $\mathrm{CO}_{2}$ counters currently in operation in our laboratory. Also the dependence of the background on atmospheric pressure (fig. 11) is comparable to that of the other counters: -2.3 percent/ cm Hg.

The presence of a possible radioactive contamination or of spurious counts due to electronic imperfections would have resulted in a lower figure. The background is not influenced by the gas purity to within 0.05 cpm. Therefore, a purity correction is not applied to the background counting rate.

An analysis of the variations of the background with time clearly shows that the counters exhibit a memory effect (fig. 12). A careful comparison of the increase in the background counting rate and the activity of the samples that filled the counters just before the anthracite revealed that the memory effect is $0.2 \%$ after 4 hours of pumping. As this is the usual pumping time for anthracite, it becomes contaminated with 0.05 cpm each time it is admitted to the counters after a modern $(250 \mathrm{cpm})$ sample. The standard for modern carbon, NBS oxalic acid, has not been affected by the memory effect to a measurable amount, as it was only admitted to the counter following modern samples that very nearly have the same activity. 


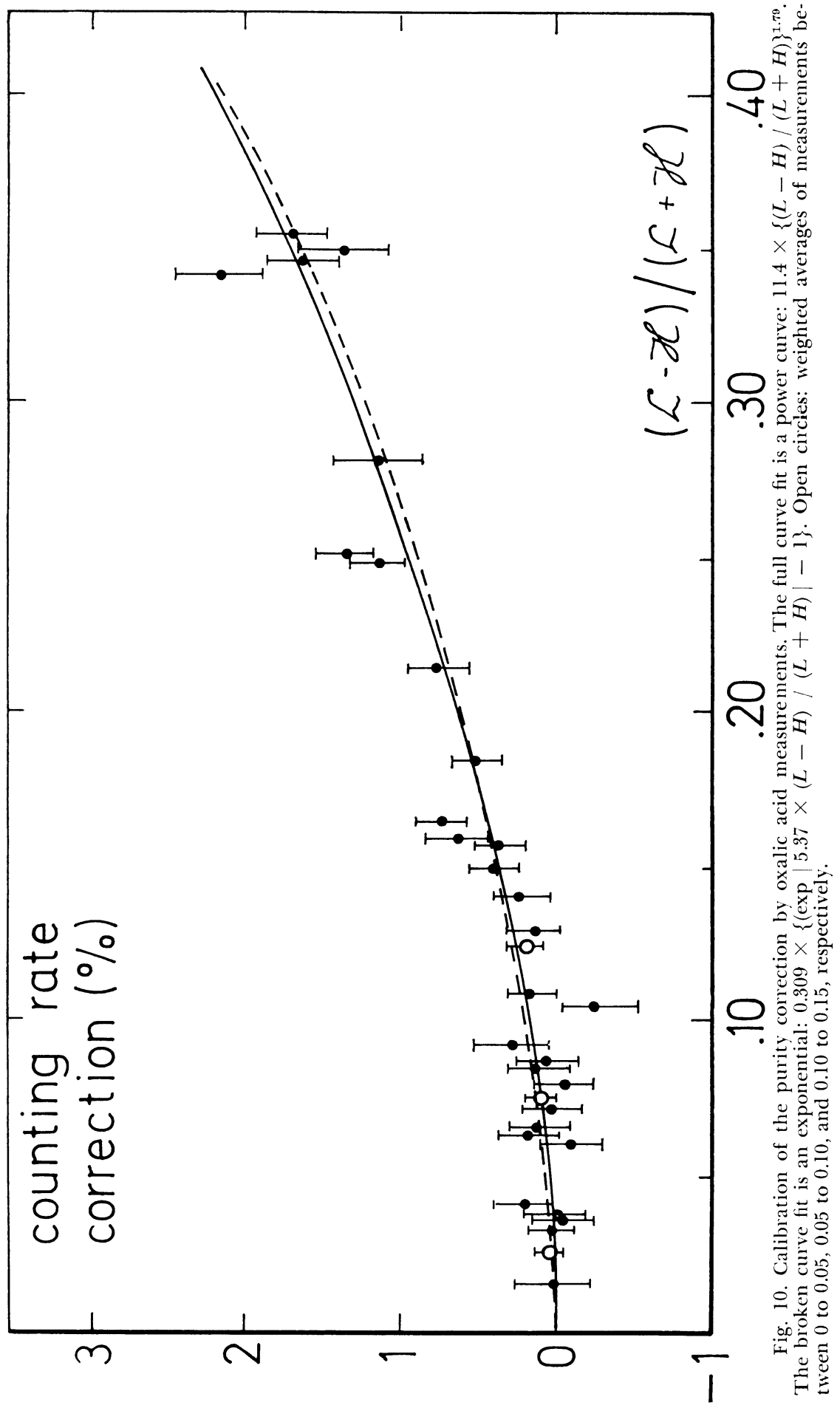




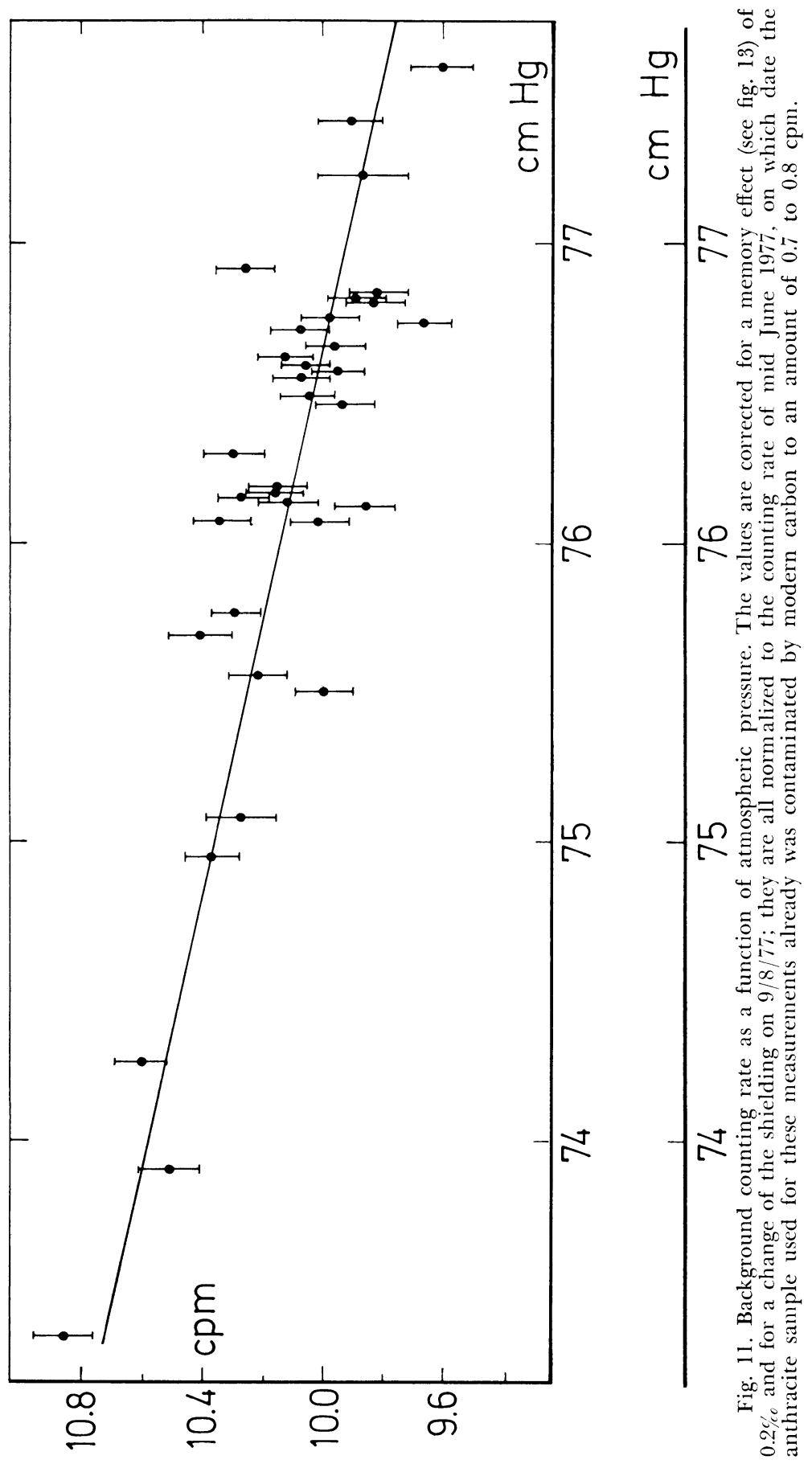




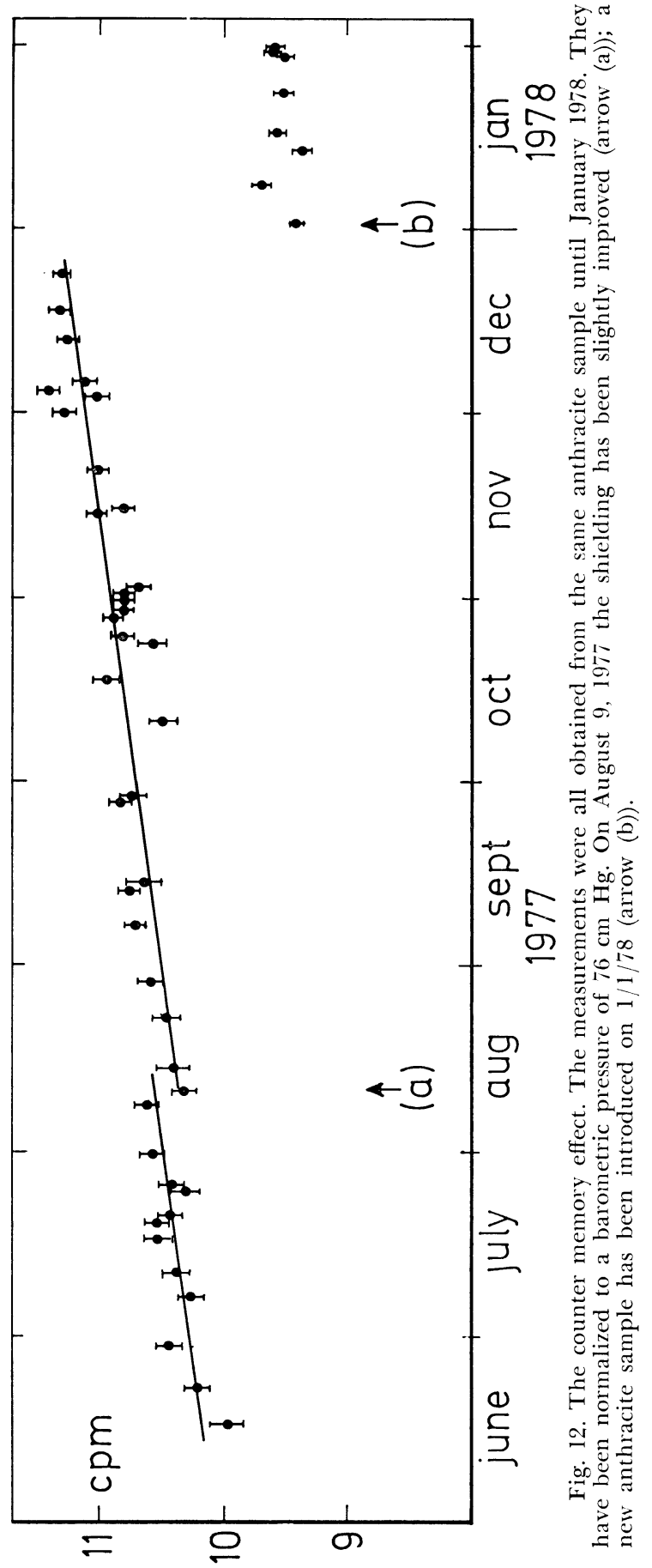




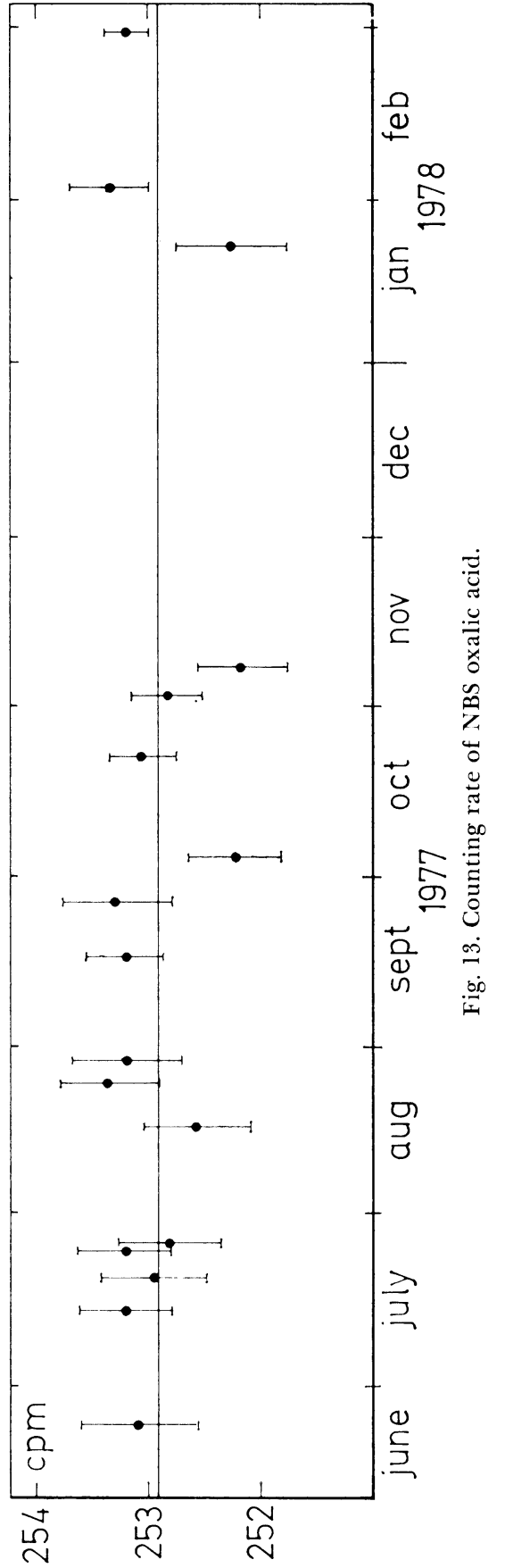


Summary of corrections applied to the measured activity.-The raw counting rate is first normalized to a "gas purity" zero (fig. 10). The background that is to be subtracted from the counting rate is corrected for a deviation from $76 \mathrm{~cm} \mathrm{Hg}$ of the average barometric pressure prevailing during the measurement (fig. 11).

After the GM counters and the proportional counters have been triggered, the system is inaccessible to a new pulse for some time (fig. 7). The total dead time is about 4 percent. The GM counting rate varies with the barometric pressure. As a result the dead time is not constant but depends both on the specific ${ }^{14} \mathrm{G}$ activity of the sample and on the barometric pressure. Corrections for this variable dead time are applied.

The ${ }^{14} \mathrm{C}$ counting rate is then normalized to a standard density of the filling gas of 0.2381 mole $/ l$. The density correction is calculated from the temperature and pressure of the filling gas, treating $\mathrm{CO}_{2}$ as a Van der Waals gas.

All samples are corrected for fractionation by normalization to a $\delta^{13} \mathrm{C}_{\mathrm{PDB}}$ value of $-25 \%$, except oxalic acid which is normalized to $\delta^{13} \mathrm{C}=$ $-19 \%$. In addition, the measured activity of oxalic acid is age-corrected to 1950 , using $\mathrm{T}_{1 / 2}=5730$ yrs.

System performance.-The operating characteristics of the system are summarized in table 1. Although it has been designed for accurate measurements of modern samples, the conventional $(2 \sigma)$ age limit of the setup is $61,500 \mathrm{yrs}$, if care is taken to measure an uninterrupted series of old samples. A memory effect of $0.2 \%$ gives an apparent age of $57,000 \mathrm{yrs}$ to anthracite measured once following a modern sample. Although it is of no importance for very old samples, the purity correction is not correct in those cases, because it applies only to the total pulse height spectrum of modern samples (mainly ${ }^{14} \mathrm{C}$ ). After having applied all corrections mentioned above, the overall accuracy for modern carbon in two counting periods of 22 hrs each, is $1.3(5) \%$, including the maximum error due to the uncertainty in the purity correction $(0.4 \%)$.

TABLE 1

System characteristics

Net recent counting rate $\left(A_{0}\right)$

$(0.95 \times$ activity NBS oxalic acid $)$

Background $(76 \mathrm{~cm} \mathrm{Hg})$

dependence on barometric pressure

Active length of each counter

Diameter of counter

Diameter of anode wire

Total active volume

Volume gas handling system

Working pressure at $24^{\circ} \mathrm{C}$

Working voltage

Gas amplification

Figure of merit

Age limit of detection (2 days)

Overall accuracy (2 days)

Capacity

$\begin{array}{cl}240.28 \pm 0.11 & \mathrm{cpm} \\ 9.40 \pm 0.05 & \mathrm{cpm} \\ 0.22 & \mathrm{cpm} / \mathrm{cm} \mathrm{Hg} \\ 74 & \mathrm{~cm} \\ 41 & \mathrm{~mm} \\ 25 & \mu \mathrm{m} \\ 6840 & \mathrm{ml} \\ 630 & \mathrm{ml} \\ 4303 & \text { torr } \\ 6750 & \text { volt } \\ 9500 & \\ 78.4 & \\ 61500 & \text { yr } \\ 1.3 & \% o \\ 125 & \text { samples } / \mathrm{yr}\end{array}$


The final test for the stability of operation of the system is the repeated measurement of NBS oxalic acid. From Figure 11, it is seen to be satisfactory.

In addition, the new set-up has been compared by making several cross checks on the same NBS oxalic acid sample with three other ${ }^{14} \mathrm{C}$ counters currently in operation in our laboratory, providing an average result of $100.06 \pm 0.28$ percent. Several measurements of ANU sucrose in the high-precision set up produced a final result of $\Delta^{14} \mathrm{C}=500.6 \pm 0.8 \%$.

With a background and an oxalic acid measurement once a week, the theoretical capacity of the system is 125 samples per year.

\section{ACKNOWLEDGMENTS}

The operation of the small high-pressure counter has thoroughly been discussed with C A M Brenninkmeyer. Additional technical details, included in this paper, have been provided by A F M de Jong, after the first author published his thesis. The oxalic acid cross crecks with other ${ }^{14} \mathrm{C}$ counters were performed by $\mathrm{H} \mathrm{J}$ Streurman. The project has been supported financially by the Netherlands Organization for Pure Scientific Research (ZWO).

\section{REFERENCES}

Bremminkmeyer, C A M, and Mook, W G, 1979, The effect of electronegative impurities on CO. proportional counting: an on-line purity test counter, in 9 th internatl conf radiocarbon dating Proc, California, June 20-26, 1976, (in press).

Groeneveld, D J, 1977, Tritium analysis of environmental water: Thesis, Univ of Groningen.

Grootes, P M, 1977, Thermal diffusion isotopic enrichment and radiocarbon dating beyond 50,000 years BP: Thesis, Univ of Groningen.

Herzenberg, A, 1969, Attachment of slow electrons to oxygen molecules: Jour Chem Phys, v 51, p. 4942-4950.

De Jong, A F M, Becker, B, and Mook, W G, 1979, Confirmation of "Suess wiggles": Nature, (in press).

Rossi, B B, and Staub, H H, 1949, Ionisation chambers and counters: McGraw-Hill Spence, D and Schulz, G J, 1972, Three body attachment in $\mathrm{O}_{2}$ using electron beams: Phys Rev, v A5, p 724-732.

Tans, P P, 1978, Carbon 13 and carbon 14 in trees and the atmospheric $\mathrm{CO}_{2}$ increase: Thesis, Univ of Groningen. 